\title{
Efeito do extrato aquoso de diferentes espécies vegetais no manejo de Meloidogyne incognita em tomateiro em ambiente protegido
}

Renato Zapparoli Corbani ${ }^{1}$

Fábio Mazzonetto²

\section{Resumo}

Em olerícolas, as perdas anuais causadas por nematoides são estimadas em cerca de 10 a 12\%. O objetivo deste trabalho foi avaliar o efeito de diferentes extratos aquosos no manejo de $\mathrm{Me-}$ loidogyne incognita em tomateiro cultivado em ambiente protegido. Foram adotados os seguintes tratamentos: testemunha (aplicação de água destilada); extrato aquoso de amora (Morus nigra) a 20\%; extrato aquoso de erva de Santa Maria (Chenopodium ambrosioides) a 20\%; extrato aquoso de capim limão (Cymbopogon citratus) a 20\%; extrato aquoso de eucalipto (Corymbia citriodora) a 20\%. Mudas de tomate cultivar Santa Clara foram transplantadas em vasos plásticos com capacidade de cinco litros. Logo em seguida, o solo foi infestado com uma suspensão contendo 5.000 ovos de $M$. incognita. No mesmo dia da infestação do solo, $25 \mathrm{~mL}$ dos extratos foram aplicados separadamente em cada vaso diretamente no solo com auxílio de uma pipeta. Para o tratamento testemunha, foram aplicados $25 \mathrm{~mL}$ de água destilada. Foram realizadas quatro aplicações a cada 15 dias. Aos 60 dias após a inoculação, avaliou-se o número de ovos e de galhas por sistema radicular, a massa fresca do sistema radicular e da parte aérea e a altura das plantas. 0 delineamento estatístico utilizado foi o inteiramente casualizado, com cinco tratamentos e sete repetições. Pode-se concluir que os extratos aquosos de amora, eucalipto, erva de Santa Maria e capim limão foram eficazes no controle de Meloidogyne incognita em tomateiro, reduzindo o número de galhas e de ovos formados.

Palavras-chave: Extrato aquoso. Meloidogyne incógnita. Solanum lycopersicum.

\section{Introdução}

Aos fitonematoides são atribuídas perdas anuais médias de cerca de $12 \%$ na maioria de nossas culturas (SASSER; FRECKMAN, 1987). Em olerícolas as perdas anuais causadas por nematoides são estimadas em cerca de 10 a 12\% (SASSER; FRECKMAN, 1987).

O controle desses patógenos é difícil e muitas vezes oneroso. Contudo, a almejada sustentabilidade do sistema produtivo requer o contínuo monitoramento desses patógenos nas áreas de cultivo da propriedade e a adoção de práticas de manejo, tanto para reduzir perdas atuais quanto para prevenir novos focos (SANTOS, 1995).

1 Universidade Camilo Castelo Branco - UNICASTELO, Professor Assistente. Descalvado, São Paulo, Brasil. renatozapparoli@ hotmail.com. (019) 35938500. Avenida Hilário da Silva Passos, 950, Bairro Parque Universitário, Descalvado, São Paulo, CEP 13690000 .

2 Universidade Camilo Castelo Branco - UNICASTELO, Professor Titular. Descalvado, São Paulo, Brasil. coord.agronomia.des@ unicastelo.br. (019) 35938500. Avenida Hilário da Silva Passos, 950, Bairro Parque Universitário, Descalvado, São Paulo, CEP 13690000. 
O manejo de nematoides empregando nematicidas usualmente é caro. Além disso, esses produtos são proibidos para a maioria de nossas culturas. Nas áreas urbanas, o emprego desses produtos para o controle de nematoides em parques e jardins não é permitido. Os nematoides das galhas têm se tornado um dos principais problemas enfrentados no cultivo de olerícolas e são responsáveis por perdas importantes, uma vez que reduzem a quantidade e a qualidade do produto colhido (SANTOS, 1995).

O uso de extratos vegetais com propriedades nematicidas no controle de fitonematoides representa mais uma alternativa para pequenos produtores, pois são práticos, custam menos que os nematicidas convencionais e representam baixo risco ao meio ambiente. Várias pesquisas têm demonstrado efeito nematicida dos extratos de diferentes plantas quando aplicados diretamente no solo (CHITWOOD, 2002)

Inúmeras são as pragas e os patógenos que causam redução de produtividade no tomateiro (Solanum lycopersicum L.), em especial os nematoides. Em âmbito global, considerando diversas espécies cultivadas, os prejuízos causados pelo ataque de nematoides chegam a 100 bilhões de dólares anuais (SASSER; FRECKMAN, 1987).

Só no Brasil, existem 43 espécies de fitonematoides em 21 gêneros associados à cultura do tomateiro (KUROZAWA; PAVAN, 2005).

Plantas de tomateiro, quando cultivadas em uma mesma área sem que medidas preventivas de controle sejam utilizadas, muitas vezes não sobrevivem ao intenso ataque da maioria das espécies de nematoide-das-galhas (Meloidogyne spp.), dependendo da infestação da área, da espécie de nematoide, da cultivar plantada e das condições ambientais (PINHEIRO et. al., 2009).

Este trabalho teve por objetivo avaliar o efeito de extrato aquoso de amora, capim limão, erva de Santa Maria e eucalipto no manejo de Meloidogyne incognita (CHITWOOD, 2002) em tomateiro em ambiente protegido.

\section{Material e métodos}

O experimento foi instalado em agosto de 2012, em casa de vegetação do Laboratório de Fitotecnia do curso de agronomia da Universidade Camilo Castelo Branco, campus Descalvado, São Paulo, Brasil. $O$ inóculo de $M$. incognita foi obtido de uma população pura, multiplicada em tomateiros, mantidos em casa de vegetação.

Os extratos aquosos de amora (Morus nigra), capim-limão (Cymbopogon citratus), erva de Santa Maria (Chenopodium ambrosioides) e eucalipto (Corymbia citriodora) foram preparados a partir da pesagem e trituração de 20 gramas de folhas secas, as quais foram mantidas em estufa de circulação forçada a $40^{\circ}$ por 48 horas para realizar a secagem, e posteriormente colocadas em suspensão em $100 \mathrm{~mL}$ de água destilada, por agitação durante 15 minutos, seguido de repouso por 24 horas e posteriormente filtrada em tecido (Voil). Os extratos foram acondicionados em recipientes de vidro âmbar e utilizados logo em seguida.

As mudas de tomateiro Santa Clara foram transplantadas em vasos plásticos com capacidade de cinco litros, contendo uma mistura de solo e areia na proporção de $1: 2(\mathrm{v}: \mathrm{v})$, previamente esterilizado em autoclave por três horas a $121^{\circ} \mathrm{C}$ e 1 atm de pressão. Em seguida, o solo de todos os vasos foi infestado com uma suspensão de $10 \mathrm{~mL}$ contendo 500 ovos $/ \mathrm{mL}$ de $M$. incognita, aplicados diretamente nas raízes. Os ovos foram extraídos seguindo o método descrito por Boneti e Ferraz (1981).

No mesmo dia da infestação do solo, $25 \mathrm{~mL}$ dos extratos foram aplicados em cada vaso, diretamente no solo, de maneira uniforme com auxílio de uma pipeta. Para o tratamento testemunha, foram aplicados $25 \mathrm{~mL}$ de água destilada. As aplicações foram feitas a cada 15 dias, totalizando quatro aplicações. 
Foram adotados os seguintes tratamentos:

a) Tratamento 1: Testemunha (aplicação de água destilada);

b) Tratamento 2: Extrato aquoso de amora a 20\%;

c) Tratamento 3: Extrato aquoso de erva de Santa Maria a 20\%;

d) Tratamento 4: Extrato aquoso de Capim Limão a 20\%;

e) Tratamento 5: Extrato aquoso de Eucalypto a $20 \%$.

Aos 60 dias após a inoculação, avaliou-se o número de ovos e de galhas por sistema radicular, massa fresca do sistema radicular e da parte aérea e a altura das plantas.

0 delineamento estatístico utilizado foi o inteiramente casualizado, com cinco tratamentos e sete repetições. Os dados obtidos foram submetidos à análise de variância pelo teste " $F$ ", a $5 \%$ de probabilidade, e as médias comparadas pelo teste de Tukey, ao nível de $1 \%$ de probabilidade.

\section{Resultados e discussão}

A aplicação dos extratos de amora, eucalipto, erva de Santa Maria e capim-limão no solo não influenciou significativamente na altura e na massa da parte aérea dos tomateiros (Tabela 1). Com relação às comparações entre as médias da massa fresca de raízes (gramas) nos diferentes tratamentos testados, os dados indicam que não houve diferença estatística entre os extratos aquosos testados com a testemunha. Entretanto, entre os extratos aquosos testados, houve diferença estatística somente entre os extratos de erva de Santa Maria e eucalipto: o extrato de erva de Santa Maria apresentou maior peso fresco de raízes (2,63 gramas) (Tabela 1).

Tabela 1. Comparação entre as médias da massa fresca da parte aérea (gramas), altura de plantas (centímetros) e massa fresca das raízes (gramas) de plantas de tomateiro, aos 60 dias após a inoculação de Meloidogyne incognita, relativo ao efeito dos extratos aquosos de amora, eucalipto, erva de Santa Maria e capim limão. Descalvado, 2012.

\begin{tabular}{cccc}
\hline Tratamentos & $\begin{array}{c}\text { Massa fresca da parte } \\
\text { aérea (gr) }\end{array}$ & $\begin{array}{c}\text { Altura das plantas (cm) } \\
\mathbf{1}^{1}\end{array}$ & $\begin{array}{c}\text { Massa fresca das } \\
\text { raízes (gr) }^{1}\end{array}$ \\
\hline Testemunha & $1,87 \mathrm{a}$ & $3,43 \mathrm{a}$ & $2,13 \mathrm{ab}$ \\
Amora & $2,39 \mathrm{a}$ & $3,59 \mathrm{a}$ & $2,32 \mathrm{ab}$ \\
Eucalipto & $2,40 \mathrm{a}$ & $3,45 \mathrm{a}$ & $2,11 \mathrm{~b}$ \\
Erva de Santa Maria & $2,62 \mathrm{a}$ & $3,67 \mathrm{a}$ & $2,63 \mathrm{a}$ \\
Capim Limão & $2,25 \mathrm{a}$ & $3,57 \mathrm{a}$ & $2,47 \mathrm{ab}$ \\
\hline Teste F & $2,07 \mathrm{NS}$ & $2,41 \mathrm{Ns}$ & $3,22^{*}$ \\
DMS (Tukey 1 \%) & 0,7929 & 0,2706 & 0,5078 \\
CV (\%) & 22,21 & 4,93 & 14,07 \\
\hline
\end{tabular}

${ }^{1}$ As médias seguidas pelas mesmas letras nas colunas não diferem estatisticamente entre si, pelo teste de Tukey, a $1 \%$.

NS Não significativo.

*significativo a 5\%. Dados transformados em DLOG $(x+1,0)$.

Fonte: Elaboração própria.

Com o objetivo de avaliar a atividade nematicida de extratos aquosos de sementes de Crotalaria breviflora, C. juncea, C. mucronata e C. spectabilis sobre Meloidogyne javanica (Treub) (CHI- 
TWOOD, 2002), em vasos de dois litros em plantas de tomateiro, Gardiano et al. (2010) observaram que os extratos de $C$. breviflora e $C$. spectabilis aumentaram respectivamente em 38,1 e $56 \%$ a massa das raízes em comparação com a testemunha infectada com o nematoide e que o número de galhas foi reduzido em 33\% apenas pela aplicação de C. mucronata.

Gardiano (2008), testando seis tinturas vegetais no controle de Meloidogyne javanica em tomateiro, produzidas com folhas de nim (Azadirachta indica), guiné (Petiveira alliacea), cinamomo (Melia azedarach), falso-boldo (Plectranthus barbatus), feijão-de-porco (Canavalia ensiformis) e mamona (Ricinus communis), pulverizadas apenas na parte aérea das plantas, sem que houvesse contato do produto com o solo, não observou efeito significativo no controle do nematoide.

Os dados relativos à comparação entre as médias do número de galhas de $M$. incognita formados por sistema radicular e número de ovos extraídos do sistema radicular, nos diferentes tratamentos testados, estão inclusos na Tabela 2. Todos os tratamentos testados diferiram estatisticamente da testemunha, apresentando menor número de galhas e de ovos formados. Não houve diferenças entre os extratos aquosos testados (Tabela 2).

Tabela 2. Comparação entre as médias do número de galhas formadas e o número de ovos extraídos de plantas de tomateiro, aos 60 dias após a inoculação de Meloidogyne incognita, demonstrando o efeito dos extratos aquosos de amora, eucalipto, erva de Santa Maria e capim-limão. Descalvado, SP, 2012.

\begin{tabular}{ccc}
\hline Tratamentos & Número de Galhas $^{1}$ & No $^{0}$ ovos $^{1}$ \\
\hline Testemunha & $3,92 \mathrm{a}$ & $5,97 \mathrm{a}$ \\
Amora & $2,30 \mathrm{~b}$ & $2,46 \mathrm{~b}$ \\
Eucalipto & $2,47 \mathrm{~b}$ & $2,53 \mathrm{~b}$ \\
Erva de Santa Maria & $2,46 \mathrm{~b}$ & $3,37 \mathrm{~b}$ \\
Capim Limão & $2,62 \mathrm{~b}$ & $3,55 \mathrm{~b}$ \\
\hline Teste F & $4,90 * *$ & $16,40 * *$ \\
DMS (Tukey 1 \%) & 1,2215 & 1,4410 \\
CV (\%) & 28,63 & 26,03 \\
\hline
\end{tabular}

${ }^{1}$ As médias seguidas pelas mesmas letras nas colunas não diferem estatisticamente entre si, pelo teste de Tukey, a $1 \%$.

**significativo a $1 \%$. Dados transformados em DLOG $(x+5,0)$.

Fonte: Elaboração própria.

Steffen (2007), testando dez diferentes óleos essenciais de plantas medicinais in vitro sobre M. graminicola, patógeno de arroz, observou que todos os óleos reduziram a eclosão de juvenis de segundo estádio do nematoide de 16 a 48\% em relação à testemunha. Dias et al. (2000) avaliaram o extrato de hortelã em juvenis de $M$. incognita e observaram baixa atividade nematicida, embora tenham registrado, também, efeito nematostático.

Gardiano et al. (2009), avaliando a adição de $20 \mathrm{~mL}$ ao solo de extratos aquosos de 20 espécies de plantas sobre a população de Meloidogyne javanica em plantas de tomateiro em casa de vegetação, verificou que os extratos de hortelã, bardana e mamona reduziram o número de galhas em $75,6 \%, 65,7 \%$ e $54,4 \%$, e o número de ovos em $81,7 \%, 75,9 \%$ e $56,6 \%$, respectivamente. 


\title{
4. Conclusões
}

Os extratos aquosos de amora, eucalipto, erva de Santa Maria e capim limão foram eficazes no controle de Meloidogyne incognita em tomateiro, reduzindo o número de galhas e de ovos formados.

\section{Effect of Aqueous Extract of Different Vegetal Species in Management of Meloidogyne incognita in Tomato in Protected Environment}

\begin{abstract}
The annual losses in vegetables caused by nematodes are estimated at around $10-12 \%$. The aim of this study was to evaluate the effect of different aqueous extracts in the management of Meloidogyne incognita on tomato cultivated in protected environment. We used the following treatments: control (treated with distilled water), aqueous extract of mulberry (Morus nigra) to 20\%, aqueous extract of herb Santa Maria (Chenopodium ambrosioides) to $20 \%$, aqueous extract of lemon grass (Cymbopogon citratus) to $20 \%$, aqueous extract of eucalyptus (Corymbia citriodora) to $20 \%$. Seedlings of tomato "Santa Clara" cultivar were transplanted into five liters plastic pots. Shortly afterwards, the soil was infested with a suspension containing 5,000 eggs of $M$. incognita. On the day of infestation of the soil, $25 \mathrm{~mL}$ of the extracts were applied separately to each vessel to the soil with a pipette. For the control, were applied $25 \mathrm{~mL}$ of distilled water was applied. There were four applications every 15 days. At 60 days after inoculation, we assessed the number of eggs and galls per root system, the fresh weight of root and shoot and plant height. The statistical design was completely randomized with five treatments and seven replicates. It can be concluded that the aqueous extract of Morus nigra, Chenopodium ambrosioides, Cymbopogon citratus and Corymbia citriodora were effective in controlling $M$. incognita in tomato by reducing the number of formed galls and egg.

Keywords: Aqueous extract. Meloidogyne incógnita. Solanum lycopersicum.
\end{abstract}

\section{Referências bibliográficas}

BONETI, J. I. S.; FERRAZ, S. Modificação do método de Hussey \& Barker para extração de ovos de Meloidogyne exigua de raízes de cafeeiro. Fitopatologia Brasileira, Brasília, DF, v. 6, n.3, p. 553, 1981.

CHITWOOD, D. J. Phytochemical based strategies for nematode control. Annual Review of Phytopathology, Palo Alto, v. 40, p. 221-249, set. 2002.

DIAS, C. R. et al. Efeito de extratos aquosos de plantas medicinais na sobrevivência de juvenis de Meloidogyne incognita. Nematologia Brasileira, Brasília, v. 24, n. 2, p. 203-210, 2000. Disponível em:<http://docentes.esalq.usp.br/sbn/nbonline/ol\%20242/203-210\%20pb.pdf > Acesso em: 21 maio 2013

GARDIANO, C.G. Pulverização de tinturas vegetais em tomateiros para o controle de Meloidogyne javanica. Revista Trópica: Ciências Agrárias e Biológicas, Chapadinha, v. 2, n. 3, p. 22, 2008. Disponível em: < http://www.ccaa.ufma.br/revistatropica/artigos_vol2_nr3/pulv_tintur_agro_art.pdf $>$ Acesso em: 21 mai. 2013. 
GARDIANO, C. G. et al. Atividade nematicida de extratos de sementes de espécies de Crotalaria sobre Meloidogyne javanica. Revista Trópica: Ciências Agrárias e Biológicas, Chapadinha, v. 4, n. 1, p. 4, 2010. Disponível em: <http://www.periodicoseletronicos.ufma.br/index.php/ccaatropica/ article/view/76 > Acesso em: 21 mai. 2013.

GARDIANO, C.G. et al. Avaliação de extratos aquosos de várias espécies vegetais, aplicados ao solo, sobre Meloidogyne javanica. Semina: Ciências Agrárias, Londrina, v. 30, n. 3, p. 551-556, jul./set. 2009. Disponível em: <http://www.uel.br/portal/frm/frmOpcao.php?opcao=http://www.uel. br/revistas/uel/index.php/semagrarias > Acesso em 21 mai. 2013.

KUROZAWA, C.; PAVAN, M. A. Doenças do tomateiro (Lycopersicon esculentum). In: KIMATI, H. et al. (Eds.) Manual de Fitopatologia: doenças das plantas cultivadas. São Paulo: Ceres, 2005, p.607-626.

PINHEIRO, J. B. et al. Identificação de fontes de resistência ao nematoide em acessos de tomateiro (Secção) Meloidogyne mayaguensis em acessos de tomateiro (Solanum Secção Lycopersicon). Brasília: Embrapa Hortaliças, 2009. (Boletim de Pesquisa e Desenvolvimento 56) Disponível em: <http://www.cnph.embrapa.br/paginas/serie_documentos/publicacoes2009/ bpd_56.pdf >. Acesso em: 21 mai. 2013.

SASSER, J. N.; FRECKMAN, D. W. A world perspective on nematology: the role of society. In: VEECH, A. J.; DICKSON, W. D. Vistas on nematology. DeLeon Springs: Society of Nematologists, 1987, p.7-14.

SANTOS, H. S. Efeitos de sistemas de manejo do solo e de métodos de plantio na produção de alface (Lactuca sativa L.) em abrigo com solo naturalmente infestado com Meloidogyne javanica. 1995. 88p. Tese (Doutorado). Universidade Federal de Lavras, Lavras.

STEFFEN, R.B. Caracterização, controle alternativo e reprodução de Meloidogyne graminicola em cultivares de arroz irrigado submetidos a diferentes regimes de umidade. 2007. 96p. Dissertação (Mestrado). Centro de Ciências Rurais, Universidade Federal de Santa Maria, Santa Maria.

\section{Histórico editorial}

Recebido: 20/02/2013

Avaliação e copidesque: 27/02/2013 a 11/06/2013 